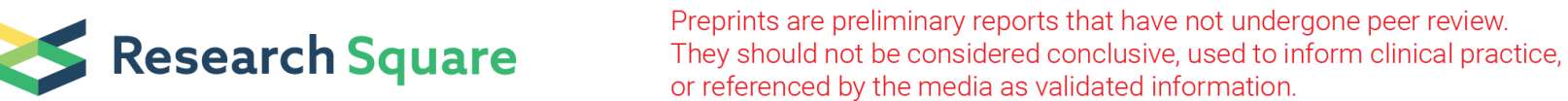

\section{Thermal-Induced Dielectric Response In Mechanically Durable Polyvinylidene Fluoride/Tubular Kapok Fiber Encapsulated Polyethylene Glycol Composite Films}

\author{
Wenqi Wu \\ Wuhan Textile University \\ Xianglin Pei \\ Guizhou Normal University \\ Hairong Li \\ hubei institute of measurement and testing technology \\ Junyang Tu \\ Wuhan Textile University \\ Qiong Li \\ Wuhan Textile University \\ Weijie Zhou \\ Wuhan Textile University \\ Yutie Gong \\ Wuhan Textile University \\ Ming Jiang ( $\nabla$ mjiang@wtu.edu.cn ) \\ Wuhan Textile University https://orcid.org/0000-0003-0415-6021 \\ Wei Gong \\ Guizhou Normal University
}

\section{Research Article}

Keywords: Dielectric response, Kapok fiber, Temperature, Polarization, Polyethylene glycol, Polyvinylidene fluoride

Posted Date: September 22nd, 2021

DOI: https://doi.org/10.21203/rs.3.rs-888235/v1

License: (c) (1) This work is licensed under a Creative Commons Attribution 4.0 International License. Read Full License 


\section{Abstract}

Dielectric materials with thermally responsive property are being pursued in fields such as next-generation sensors, smart switches, and novel actuators. These applications require that the dielectric materials have mechanical durability and stable serviceability besides thermally responsive dielectric behavior. Herein, we report a novel thermally responsive, mechanically durable, and low-cost dielectric composite simply fabricated by vacuum impregnating polyethylene glycol (PEG) into Kapok fiber (KF) and compounding them with polyvinylidene fluoride (PVDF). A remarkable dielectric susceptibility, controlled dielectric transition temperature and obvious thermal hysteresis of the composite films induced by the solid-liquid phase transition of PEG are demonstrated. The effect of molecular weights of PEG on the dielectric response behaviors is evaluated. Such thermally responsive dielectric materials with satisfactory mechanical durability will offer a chance toward constructing thermally responsive systems for safe and stable operation.

\section{Introduction}

Thermally responsive dielectric materials have received increasing attention in the last decade because of their ability to undergo dielectric transitions in response to temperature stimuli (Rullyani et al., 2020; Shafranek et al., 2019; Y. Wang, Zhang, \& Zhang, 2021; Yang et al., 2021). These materials can undergo transitions from high to low dielectric states or vice versa in a narrow temperature interval (Ganguly et al., 2019; Jiang et al., 2020; Li et al., 2019; Meng et al., 2017; Rok et al., 2020), and exhibit a distinct bistability (Shao, Zhang, Shi, Yao, \& Zhang, 2015; Szeremeta et al., 2021; Uezu et al., 2020), having a wide range of applications in new smart devices, sensors, switches, actuators, etc. Among these materials, porous molecular materials containing guest small molecules or ions have become a research hotspot (Xu, Zhang, Huang, \& Liu, 2021). When the temperature reaches a certain temperature, the ordered-disordered transition produced by the guest molecules within the system allows the appearance of two or more significantly different dielectric states (Uezu et al., 2020; Zhang et al., 2014). Despite the excellent dielectric properties of such materials, the complicated design and preparation process, as well as the intrinsic defects in the mechanical properties of the materials, make them a major obstacle in their applications. Moreover, the mechanical properties of thermo-responsive dielectric materials have rarely been reported. It actually is challenging to develop a simple and low-cost approach to fabrication of flexible and mechanically durable thermally responsive dielectric materials. Recently, a thermal-induced dielectric susceptibility has been observed in PEG-contained composites (Tu et al., 2019; W. Wang et al., 2020). The use of PEG as a thermally responsive dielectric material is very promising. The switching between high and low dielectric states can be triggered by the crystalline-amorphous phase transition of PEG induced by heat (Sun, Peng, Zhang, \& Zhu, 2020). However, ensuring the satisfactory mechanical properties of the materials, shape stability as well as enhanced dielectric polarization during the phase transition is a major challenge in their service processes. In recent years, Kapok fiber (KF), a cellulose material, has received tremendous attention as a renewable adsorbent and template material (Zheng, Wang, \& Wang, 2021) for its natural microtubule structure and high void content of more than $80 \%$, 
besides its other desirable properties such as low cost, biodegradability and high specific properties compared with conventional inorganic fillers. The usage of KF as a PEG carrier is expected to prevent PEG leakage, ensure the shape stability and achieve low density.

Herein, authors report the simple fabrication of a novel thermally responsive, mechanically durable, and low-cost dielectric composite using natural tubular KF as the PEG carrier, PVDF as the matrix, and PEG as the phase change material, which responds to temperature. The PVDF is utilized to enhance the dielectric polarization and strengthen mechanical property. The structure, thermal, dielectric and specific properties of the PVDF/KF/PEG composites were investigated, and the effect of the molecular weights of PEG on the dielectric response and thermal hysteresis were evaluated. The results presented in this study contribute to both basic and applied research as well as exhibit high potential for novel electronic device applications.

\section{Experimental}

\section{Materials and preparation}

The Kapok fibers used were common products that can be purchased in the market. PEG (800, 2k, 10k) was purchased from Sinopharm Group Chemical Reagent Co. PVDF was purchased from Shanghai San Aifu New Material Co. All other drugs were of analytical purity and purchased from Sinopharm Chemical Reagent Co. PEG was encapsulated into natural Kapok fibers by vacuum impregnation. The Kapok fibers were crushed into short fibers of about 0.3-0.5 mm in length and placed into a container, then an appropriate amount of dissolved PEG was added to completely submerge the Kapok fibers and placed in a vacuum for 1 hour. After the samples were dried, the samples were washed twice with DMF to remove the PEG attached to the surface. Next, a certain amount of PVDF and DMF were added to the Kapok fibers, stirred until well dispersed, and the mixture was placed in an oven and left to stand until the solvent evaporated completely. Finally, the resulting sample was hot pressed to obtain PVDF/KF/PEG composite film.

\section{Characterization}

The dielectric properties of the sample films, including conductivity, dielectric constant and dielectric loss, were tested using a dielectric spectrometer (KYKYSBC-12 LCR Co., Ltd., Japan). The scanning frequency was $100 \mathrm{~Hz}$ to $5 \mathrm{MHz}$ and the temperature range was $0-80^{\circ} \mathrm{C}$. The surface morphology of the material was characterized using field emission electron microscopy. The differential scanning calorimetry analysis was performed using a DSC3500 (NETZSCH Group, Germany) under a nitrogen atmosphere with a double-cycle test at a temperature variation rate of $10^{\circ} \mathrm{C} \cdot \mathrm{min}^{-1}$ from $0-80^{\circ} \mathrm{C}$. The chemical structure of the composite phase change energy storage material was tested using Fourier transform infrared spectroscopy with a scan interval of $4000-400 \mathrm{~cm}^{-1}$. X-ray tests were performed on an X-ray generator with a scan range of $2 \theta=5-60^{\circ}$. The microscopic morphology of the composite films was characterized by means of a field emission electron microscope (JSM-6360LV, JEOL). Thermogravimetric analysis was 
performed by an STA2500 (NETZSCH, Germany), where the samples were elevated from $20^{\circ} \mathrm{C}$ to $800^{\circ} \mathrm{C}$ at a rate of $10 \mathrm{k} \cdot \mathrm{min}^{-1}$ under a nitrogen atmosphere. The specific properties of KF/PVDF composites were obtained by stretching dumbbell samples of the composite films at a stretching rate of $20 \mathrm{~mm} \cdot \mathrm{min}^{-1}$ using an Instron stretching machine.

\section{Results And Discussion}

\section{Structural analysis of KF/PEG and PVDF/KF/PEG composite films}

Figure 1 shows the XRD patterns of KF, KF/PEG, and PVDF/KF/PEG composite films, respectively. It can be seen from Fig. 1 that the natural KF is amorphous in structure, and all the PVDF/KF/PEG show distinct characteristic peaks at $2 \theta=19.20^{\circ}$ and $2 \theta=23.35^{\circ}$, corresponding to the (120) and (112) crystal planes of PEG (Fig.S1), respectively. Moreover, no other new characteristic peaks are observed in Fig.1a, indicating the absence of chemical reactions during the impregnation of the KF with PEG. The XRD patterns of both KF/PVDF and PVDF/KF/PEG composite films (Fig.1b) display the characteristic peaks of PVDF at $2 \theta=18.24^{\circ}, 20.16^{\circ}, 26.56^{\circ}$, corresponding to (020), (110), (021) crystal plane of PVDF. And the characteristic peaks of PEG also can be found at $2 \theta=19.1^{\circ}$ and $23.6^{\circ}$. The above results show that both the compounded PEG and PVDF maintain their crystallization ability, and there is no significant change in the crystal structure.

The TG curves of KF, KF/PEG, and PVDF/KF/PEG composite films are exhibited in Figure 2. The major thermal weight loss of pure PVDF starts at $450^{\circ} \mathrm{C}$ and ends at $480^{\circ} \mathrm{C}$. The thermal weight loss of the samples reaches $60 \%$ at this stage and the total thermal weight loss up to $800^{\circ} \mathrm{C}$ is about $68 \%$. The main weight loss of PEG800, PEG2k and PEG10k start at $350^{\circ} \mathrm{C}-380^{\circ} \mathrm{C}$, and end at $420^{\circ} \mathrm{C}-440^{\circ} \mathrm{C}$, respectively. An initial mass loss of the KF is found due to the water adsorbed on the fiber surface, and the weight loss of $\mathrm{KF}$ mainly occurs in the range of $250-350^{\circ} \mathrm{C}$, which can be attributed to the degradation of a large amount of hemicellulose in KF. There is almost no weight loss of the PVDF/KF/PEG before $270^{\circ} \mathrm{C}$, and as the temperature increased from $270^{\circ} \mathrm{C}$ to $480^{\circ} \mathrm{C}$, TG curves of all the composites showed significant weight loss in function of temperature increase. The mass loss in the range of $270-350^{\circ} \mathrm{C}, 350-450^{\circ} \mathrm{C}$ and $450-480^{\circ} \mathrm{C}$ is primarily attributed to the thermal pyrolysis of the KF, PEG and PVDF, respectively. The PEG mass ratios (Table S1) were obtained by calculating the residual carbon rates of different composite films, where PVDF/KF/PEG800 adsorbed the most mass of PEG with a mass ratio of $17.3 \%$, compared to $16.6 \%$ and $10.9 \%$ for PVDF/KF/PEG2k and PVDF/KF/PEG10k, respectively.

SEM images (Figure 3) show the cross sections of the PVDF/KF/PEG composite films. The KF displays a high hollow ratio of $\sim 80 \%$, which provides sufficient space for PEG impregnation. Meanwhile, the unique hollow structure that possesses a wall thickness of around $1 \mu \mathrm{m}$ facilitates not only the reduction in the mass proportion of non-phase change components but also the improvement in the dielectric response efficiency of the composites. Figure 3 shows that the KF/PEG fibers are randomly dispersed and disoriented in the PVDF matrix, and the tubular structure of the KF fibers remains intact after the solution casting and hot pressing. After compounding the KF/PEG fiber with PVDF matrix, the solid PEG can still 
be clearly observed from the hollow cavity of the KF, and there is obvious interface debonding in the PVDF matrix and KF. This is mainly due to the PEG crystallization-induced shrinkage and the weak bond between PEG and the KF inner surface. It can also be found that the KF as the PEG carrier does not inhibit or promote the phase change of PEG.

\section{Thermal-induced phase transition in the PVDF/KF/PEG}

The phase change of the PEG in the composite films is an essential prerequisite for triggering dielectric response. Figure 4 and Figure S2 show the melting and freezing DSC curves of the KF/PEG and $P V D F / K F / P E G$. Figure S2d shows both the melting and crystallization temperatures of the KF/PEG increase as the molecular weight of the PEG increases. The melting transitions of the KF/PEG containing PEG of various molecular weights occur within the range of $14.0-24.6^{\circ} \mathrm{C}, 49.9-64.5^{\circ} \mathrm{C}$ and $51.8-65.8^{\circ} \mathrm{C}$, with peak temperatures of $19.6^{\circ} \mathrm{C}, 53.8^{\circ} \mathrm{C}$ and $61.9^{\circ} \mathrm{C}$, respectively. The molecular weight dependence of the melting and crystallization temperatures of the PVDF/KF/PEG is similar to those of the KF/PEG. In addition, the latent heats of the KF/PEG and PVDF/KF/PEG are obviously lower than that of the pure PEG due to the incorporation of the KF and/or PVDF that does not undergo phase change within the measured temperature range (Table S2).

\section{Mechanical properties of PVDF/KF/PEG composite films}

The PEG leakage during the phase transition can be effectively prevented by using the hollow tubular KF. However, the hollow structure of KF and its high moisture absorption like other cellulose fibers inevitably lead to poor mechanical properties, and ubiquitous micro-biological attack could result in further degradation in the mechanical properties and the reduction in its service life. This makes the KF/PEG difficult to address the mechanical performance requirements of electronic device applications. Therefore, the incorporation of PVDF is not only expected to enhance the dielectric polarization of the composites but also increase their mechanical strength and life span. Figure 5 shows the specific strength of the PVDF/KF films as functions of KF content. The stress-strain curves of the composite films are displayed in Figure S3. The drop in the specific strength has been caused by the increase in void content with increasing fiber loading. But even in the case of high fiber content, relatively high specific strength is attained. As we know, the macroscopic mechanical properties of such composites are determined predominantly by the properties of the fiber-matrix interface. The mechanical properties are expected to further improve via strengthening the fiber-matrix interface in consideration of the inadequate bond strength in this composite system.

\section{Dielectric properties of PVDF/KF/PEG composite film}

The effects of frequency and PEG molecular weight on the dielectric properties of the PVDF/KF/PEG composite films is shown in Figure 6 . The relative dielectric constant $\left(\boldsymbol{\varepsilon}_{r}\right)$ of nearly all specimens goes down as frequency goes up (Figure 6a). The dielectric constants of the composite film at the same frequency are significantly higher than that of the pristine PVDF, especially at low frequency range. This indicates that the polarization ability of the composite films is significantly enhanced due to the 
introduction of polar PEG of relatively small molecular weights (Yamamoto, Minami, Hui, Morikawa, \& Kimizuka, 2020). Besides, the PEG with lower molecular weight has a higher relative proportion of polar sections, and thus results in higher electric polarization under the electric field. It can be seen from Fig. $6 \mathrm{~b}$ that the loss tangents of the PVDF and the composites decrease with frequency increase and then begin to increase at frequency $\sim 10^{5} \mathrm{~Hz}$, which is typical feature of conduction electron and interfacial polarization relaxation. It is worth noting that the loss tangent values of all the composites are higher than that of PVDF. and the smaller the molecular weight of PEG, the higher the loss tangent value of the composites at the same frequency, especially in the middle and low frequency range, which is related to the introduction of polar PEG molecules. Additionally, the increased defects and interfaces inside the composites caused by the introduction of fibers contribute to the significant increase in interfacial loss.

The dielectric constants of the PVDF, PVDF/KF/PEG800, PVDF/KF/PEG2k, and PVDF/KF/PEG10k at 1k $\mathrm{Hz}$ are plotted against temperature in Fig. 7. The pure PVDF shows a gradual rise in dielectric constant with temperature. While the dielectric constants of all the composites significantly increase with temperature in the measured temperature range. The PVDF/KF/PEG800 undergo a noteworthy $\boldsymbol{\varepsilon}_{r}$ increase around $25^{\circ} \mathrm{C}$, which matches the melting temperature span of PEG800 in the composite. Both the PVDF/KF/PEG2k and PVDF/KF/PEG10k also exhibit a similar trend of the temperature dependence of dielectric constant to the PVDF/KF/PEG800. The dielectric susceptibility in the composites is temperature dependent, and the temperature intervals of the dielectric transition are roughly consistent with the melting temperature ranges of the relevant molecular weight of the PEG in the composites. The melting phase transition of PEG upon heating increases considerably the effective dipole moment that is mainly associated with orientational polarization, and thus contributes to the dramatic rise of the dielectric constant. In addition, the conductivity of the composites also showed a similar transition pattern with temperature to the dielectric constant (Figure S4). Although the temperature range of the dielectric transition does not completely consistent with that of the conductivity susceptibility, it can be inferred that conduction electron polarization partially contributes to the dielectric response. With the increase of PEG molecular weight, the temperature width of the dielectric transition of the composites gradually decreases. Among them, the dielectric transition temperature range of PVDF/KF/PEG10k is the narrowest, which may be related to the relatively high perfection degree of crystal structure of the PEG10k.

The change in polarity before and after the phase transition can result in significant difference in dielectric state, which provides the precondition for the dielectric bistability of the composites. For thermally-responsive dielectric materials, a relatively wide hysteresis loop at temperatures near room temperature is also important for their practical application besides bistability (Cao et al., 2021; Liu, Haibibu, Xu, Han, \& Wang, 2020; Tripathi et al., 2021; Yamaguchi, 2021). The structural phase transition of PEG, depending on the molecular weight, typically occurs at or near room temperature. The thermal hysteresis behavior of the PVDF/KF/PEG composites containing PEG of different molecular weights during the heating and cooling cycles is illustrated in Figure 8. The three composites exhibited different dielectric transition characteristics during heating and cooling, and the dielectric thermal hysteresis loop gradually widens as the molecular weight of PEG in the composites increases, among which the 
hysteresis loop width of PVDF/KF/PEG10k achieves $16.5^{\circ} \mathrm{C}$ (Table S3). Unlike other types of dielectric responsive materials based on inorganics or organic small molecules, PEG, as a polymer, possesses relatively long relaxation time because of the high molecular weight. The long-chain structure of PEG plays an important role in the melting behavior and crystallization kinetics caused by intermolecular friction within the PEG. The supercooling effect affected by the dynamics or relaxation of polymer chains leads to the differences in the temperature shifts of crystallization and melting, accordingly generating a dielectric hysteresis loop. The molecular chain length of the PEG plays critical role on the intermolecular actions. As the molecular weight increases, the intermolecular interactions between the PEG chains increases, leading to enhanced supercooling effect and thus the increased relaxation time.

\section{Conclusion}

A novel thermally responsive, mechanically durable, and low-cost dielectric composite simply fabricated by vacuum impregnating PEG into KF and compounding them with PVDF has been reported. The structure, thermal, dielectric and specific properties of the PVDF/KF/PEG composites were investigated, and the effect of the molecular weights of PEG on the dielectric response and thermal hysteresis were evaluated. The results reveal a remarkable dielectric susceptibility, controlled dielectric transition temperature and obvious thermal hysteresis of the composite film induced by the solid-liquid phase transition of PEG. The change in polarity before and after the phase transition induced by heat can result in significant difference in dielectric state, which facilitates the dielectric bistability of the composites. A $16.5^{\circ} \mathrm{C}$ wide hysteresis loop in the thermally-responsive dielectric composites at temperatures near room temperature is attained. Such thermally responsive dielectric materials, based on a non-ferroelectric phase transition, with satisfactory mechanical durability will offer a chance toward constructing low-cost thermally responsive systems for safe and stable operation.

\section{Declarations}

\section{Acknowledgments}

We gratefully acknowledge the supports by the National Natural Science Foundation of China (Grant No. 51503158), the Key Research Project of Science and Technology of Department of Education of Hubei Province (Grant No. D20201703) and the Open Project of Key Laboratory of Textile Fiber and Products (Ministry of Education) (Grant No. Fzxw2021008).

\section{References}

1. Cao, S., Xu, J., Jin, L., Zhao, J., Ma, Z., Chen, Q.,. .. Gao, F. (2021). Microstructure and bidirectional dielectric tunability behaviour of Nd3+-doped KSr2Nb5015 lead-free ceramics. Journal of Materiomics, 7(5), 976-987. https://doi.org/https://doi.org/10.1016/j.jmat.2021.02.016

2. Ganguly, R., Acharyya, A., Zamin, H., Ramadurai, R., Saxena, N., \& Manivannan, A. (2019). Dielectric switching studies of polyvinylidene fluoride thin films with dominant planar ferroelectric domain 
configuration for flexible electronic devices. IEEE Transactions on Dielectrics and Electrical Insulation, 26(4), 1371-1377. https://doi.org/10.1109/tdei.2019.008101

3. Jiang, J. Y., Xu, Q., Ma, J. J., Gong, Z. X., Shi, C., \& Zhang, Y. (2020). Above room-temperature dielectric switching and semiconducting properties of a layered organic-inorganic hybrid compound: (C6H12N)2Pb(NO3)4. Dalton Trans, 49(46), 16860-16865. https://doi.org/10.1039/d0dt03206a

4. Li, H. J., Liu, Y. L., Chen, X. G., Gao, J. X., Wang, Z. X., \& Liao, W. Q. (2019). High-Temperature Dielectric Switching and Photoluminescence in a Corrugated Lead Bromide Layer Hybrid Perovskite Semiconductor. Inorg Chem, 58(15), 10357-10363. https://doi.org/10.1021/acs.inorgchem.9b01538

5. Liu, Y., Haibibu, A., Xu, W., Han, Z., \& Wang, Q. (2020). Observation of a Negative Thermal Hysteresis in Relaxor Ferroelectric Polymers. Advanced Functional Materials, 30(25), 2000648. https://doi.org/https://doi.org/10.1002/adfm.202000648

6. Meng, P., Zhang, Q., Wu, Y., Tan, Z., Cheng, G., Wu, X., \& Zheng, R. (2017). Room-Temperature Dielectric Switchable Nanocomposites. Advanced Functional Materials, 27(30). https://doi.org/10.1002/adfm.201701136

7. Rok, M., Zarychta, B., Moskwa, M., Dziuk, B., Medycki, W., \& Bator, G. (2020). Structural phase transitions coupled with prominent dielectric anomalies and dielectric relaxation in $[(\mathrm{CH} 3) 3 \mathrm{NH}] 2[\mathrm{KCo}(\mathrm{CN}) 6]$ and mixed $[(\mathrm{CH} 3) 3 \mathrm{NH}] 2[\mathrm{KFexCo1}-\mathrm{x}(\mathrm{CN}) 6]$ double perovskite hybrids. Dalton Trans, 49(6), 1830-1838. https://doi.org/10.1039/c9dt04703d

8. Rullyani, C., Singh, M., Li, S.-H., Sung, C.-F., Lin, H.-C., \& Chu, C.-W. (2020). Stimuli-responsive polymer as gate dielectric for organic transistor sensors. Organic Electronics, 85, 105818. https://doi.org/https://doi.org/10.1016/j.orgel.2020.105818

9. Shafranek, R. T., Millik, S. C., Smith, P. T., Lee, C.-U., Boydston, A. J., \& Nelson, A. (2019). Stimuliresponsive materials in additive manufacturing. Progress in Polymer Science, 93, 36-67. https://doi.org/https://doi.org/10.1016/j.progpolymsci.2019.03.002

10. Shao, X. D., Zhang, X., Shi, C., Yao, Y. F., \& Zhang, W. (2015). Switching Dielectric Constant Near Room Temperature in a Molecular Crystal. Adv Sci (Weinh), 2(5), 1500029. https://doi.org/10.1002/advs.201500029

11. Sun, G., Peng, S., Zhang, X., \& Zhu, Y. (2020). Switchable Electromagnetically Induced Transparency with Toroidal Mode in a Graphene-Loaded All-Dielectric Metasurface. Nanomaterials (Basel), 10(6). https://doi.org/10.3390/nano10061064

12. Szeremeta, A. Z., Nowok, A., Pawlus, S., Fedoruk, K., Trzebiatowska, M., Mączka, M.,. .. Sieradzki, A. (2021). Stable and reversible pressure-controlled dielectric switching in dicyanide hybrid perovskite. Applied Materials Today, 22. https://doi.org/10.1016/j.apmt.2021.100957

13. Tripathi, A., John, J., Kruk, S., Zhang, Z., Nguyen, H. S., Berguiga, L.,. .. Cueff, S. (2021). Tunable MieResonant Dielectric Metasurfaces Based on VO2 Phase-Transition Materials. ACS Photonics, 8(4), 1206-1213. https://doi.org/10.1021/acsphotonics.1c00124

14. Tu, J., Li, H., Cai, Z., Zhang, J., Hu, X., Huang, J., .. Huang, L. (2019). Phase change-induced tunable dielectric permittivity of poly(vinylidene fluoride)/polyethylene glycol/graphene oxide composites. 
Composites Part B: Engineering, 173. https://doi.org/10.1016/j.compositesb.2019.106920

15. Uezu, Y., Tsunashima, R., Tanaka, C., Fujibayashi, M., Manabe, J., Nishihara, S., \& Inoue, K. (2020). Spin Crossover between the High-Spin and Low-Spin States and Dielectric Switching in the lonic Crystals of a Fe(II) [2 × 2] Molecular Grid. Bulletin of the Chemical Society of Japan, 93(12), 15831587. https://doi.org/10.1246/bcsj.20200207

16. Wang, W., Li, H., Zhao, J., Tu, J., Wu, W., Luo, Z., .. Huang, L. (2020). Dielectric Response Triggered by a Non-ferroelectric Phase Transition in Poly(Vinylidene Fluoride)/Poly(Ethylene Glycol)/Halloysite Composites. Journal of Macromolecular Science, Part B, 59(12), 867-877. https://doi.org/10.1080/00222348.2020.1809190

17. Wang, Y., Zhang, Y.-M., \& Zhang, S. X.-A. (2021). Stimuli-Induced Reversible Proton Transfer for Stimuli-Responsive Materials and Devices. Accounts of Chemical Research, 54(9), 2216-2226. https://doi.org/10.1021/acs.accounts.1c00061

18. Xu, X.-Q., Zhang, H., Huang, X.-Q., \& Liu, Y.-L. (2021). A high-temperature halide perovskite molecular ferroelastic with evident dielectric switching. Inorganic Chemistry Frontiers, 8(5), 1197-1204. https://doi.org/10.1039/d0qi01365j

19. Yamaguchi, M. (2021). Thermal Hysteresis Involving Reversible Self-Catalytic Reactions. Accounts of Chemical Research, 54(11), 2603-2613. https://doi.org/10.1021/acs.accounts.1c00090

20. Yamamoto, R., Minami, Y., Hui, J. K. H., Morikawa, M.-a., \& Kimizuka, N. (2020). Enhanced Electric Polarization and Polar Switching of Dipolar Aromatic Liquids Confined in Supramolecular Gel Networks. Journal of the American Chemical Society, 142(3), 1424-1432. https://doi.org/10.1021/jacs.9b11276

21. Yang, P., Zhu, F., Zhang, Z., Cheng, Y., Wang, Z., \& Li, Y. (2021). Stimuli-responsive polydopaminebased smart materials. Chemical Society Reviews. https://doi.org/10.1039/D1CS00374G

22. Zhang, Y., Ye, H. Y., Cai, H. L., Fu, D. W., Ye, Q., Zhang, W., .. Xiong, R. G. (2014). Switchable dielectric, piezoelectric, and second-harmonic generation bistability in a new improper ferroelectric above room temperature. Adv Mater, 26(26), 4515-4520. https://doi.org/10.1002/adma.201400806

23. Zheng, Y., Wang, J., \& Wang, A. (2021). Recent advances in the potential applications of hollow kapok fiber-based functional materials. Cellulose, 28(9), 5269-5292. https://doi.org/10.1007/s10570-021-03834-6

\section{Figures}



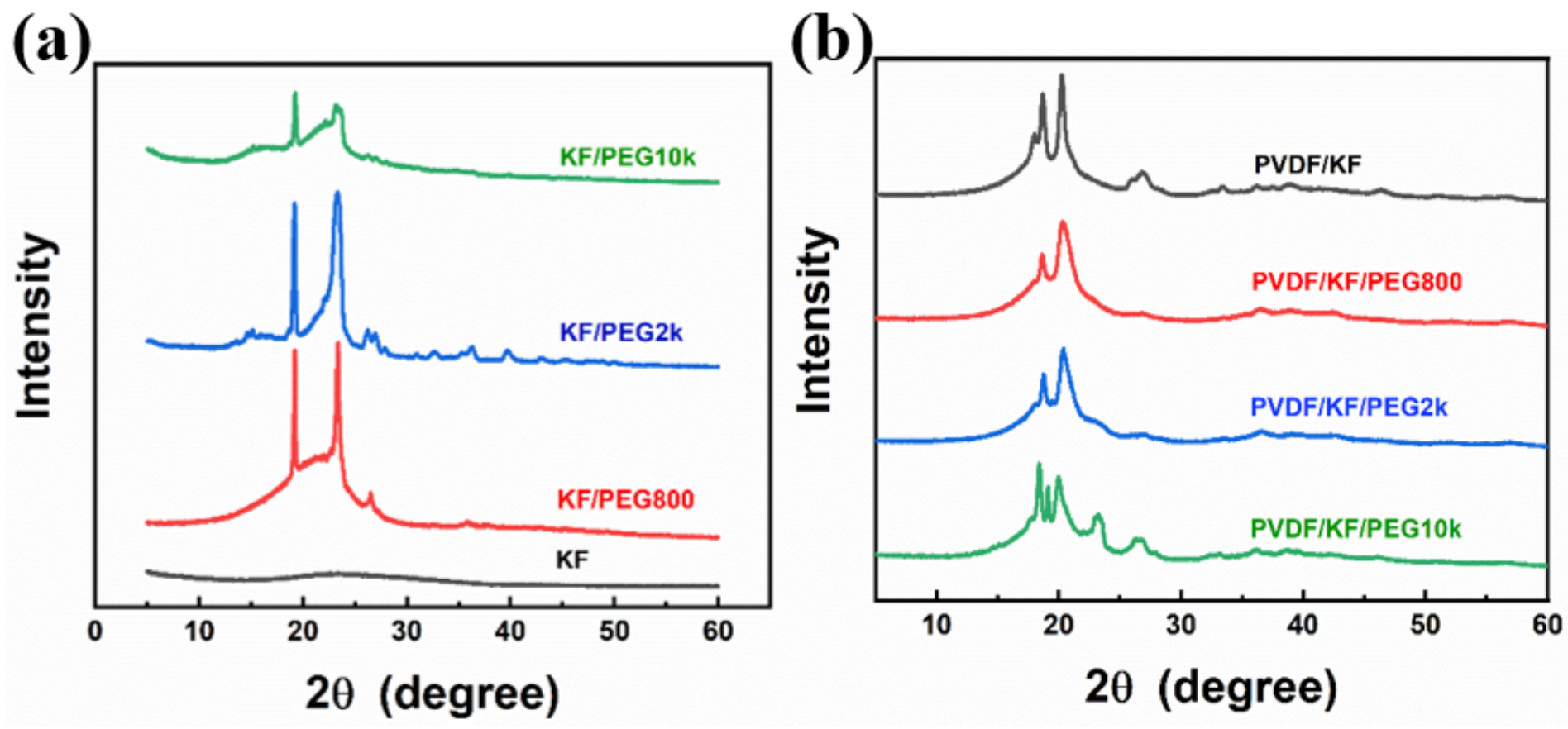

Figure 1

XRD patterns of KF/PEG (a) and PVDF/KF/PEG (b).
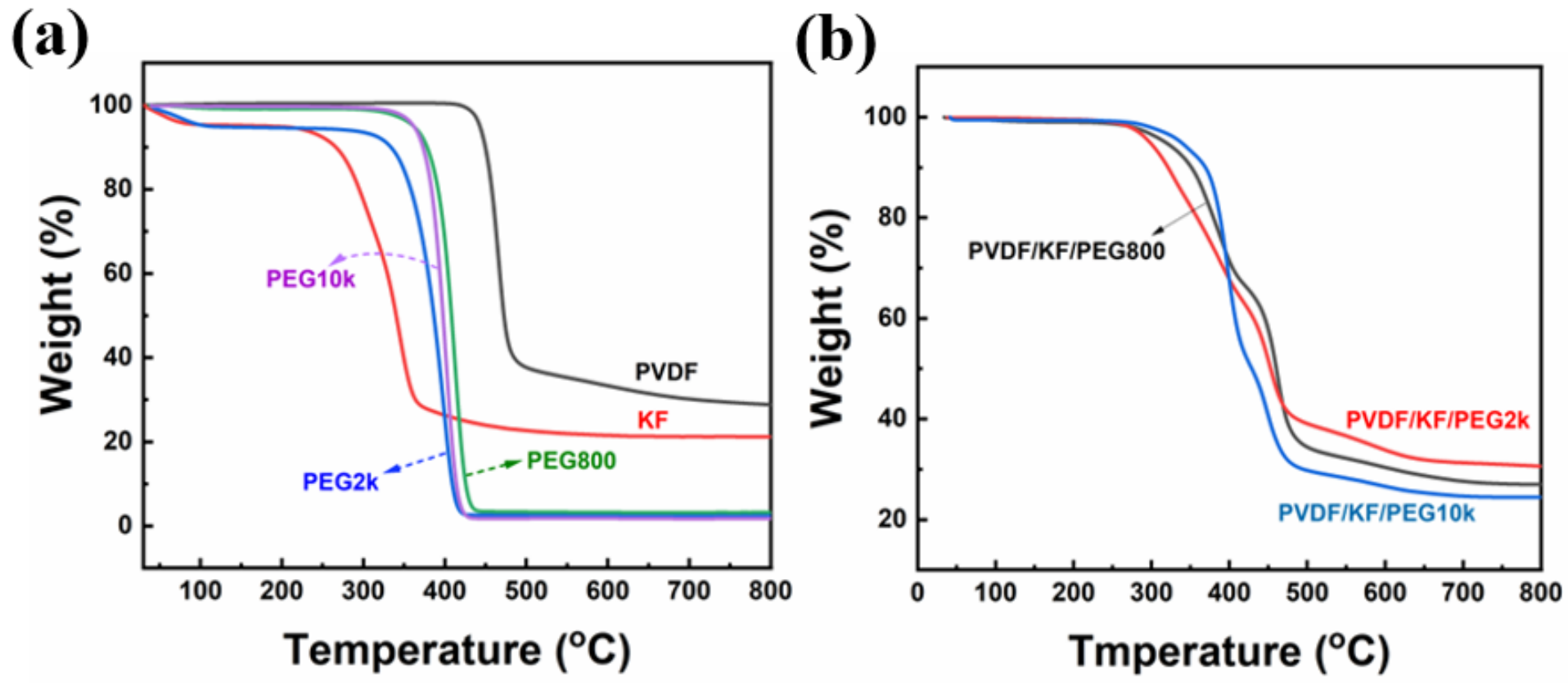

Figure 2

TG curves of KF, PVDF, PEG (a) and PVDF/KF/PEG composite films (b). 


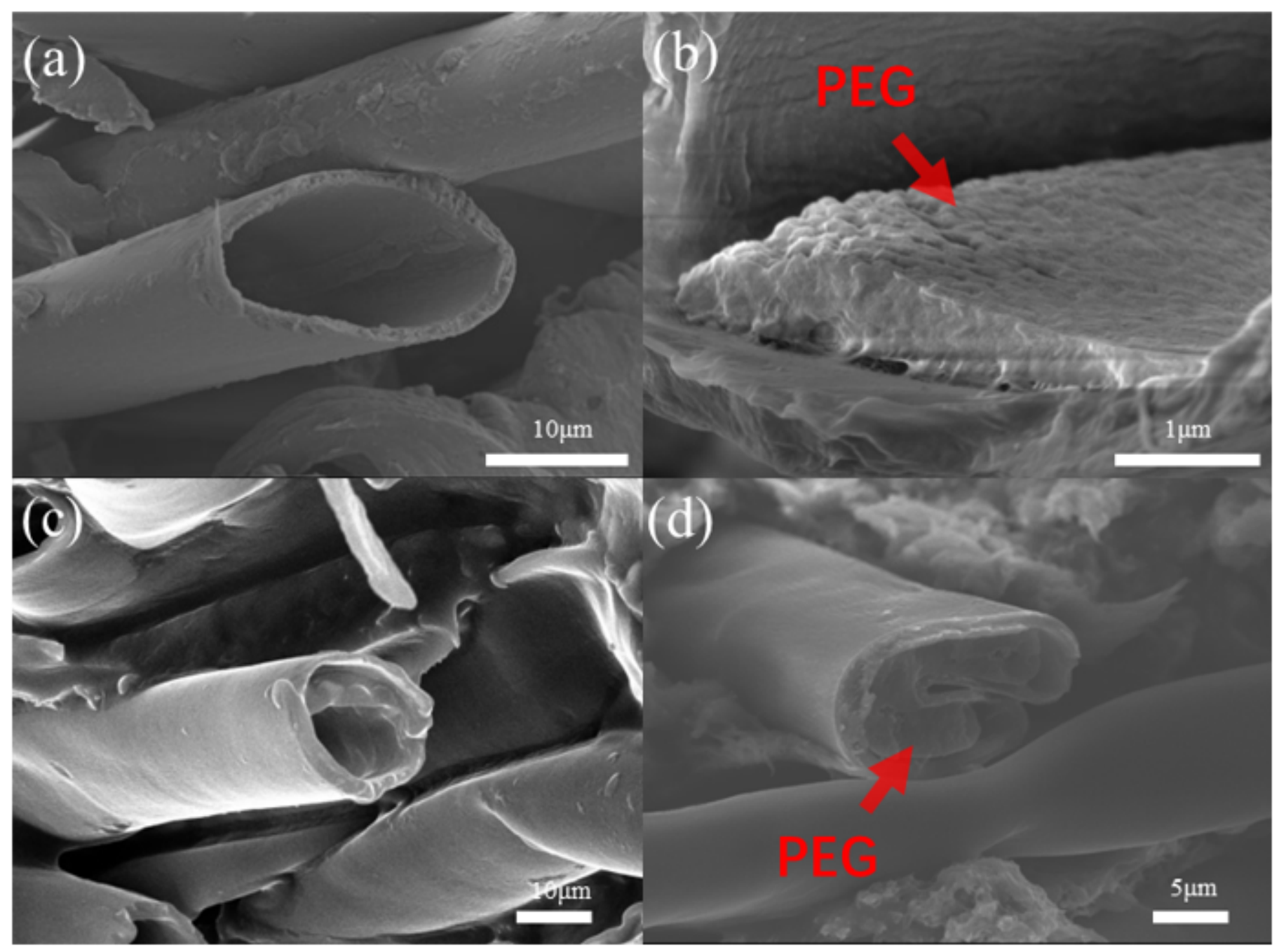

Figure 3

SEM images of KF (a), KF/PEG (b) and PVDF/KF/PEG2k (c,d). 

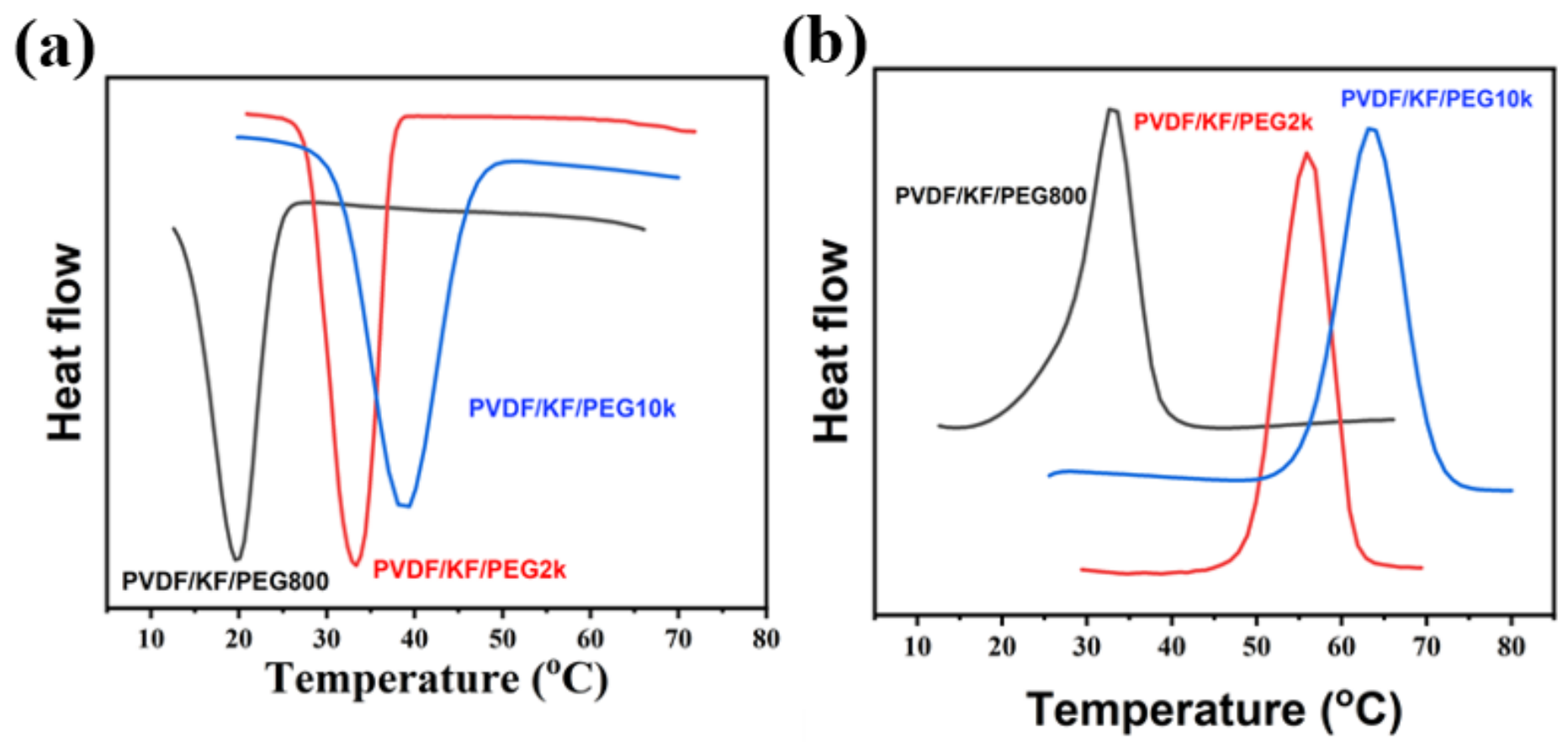

Figure 4

DSC curves of the PVDF/KF/PEG containing PEG of various molecular weights.

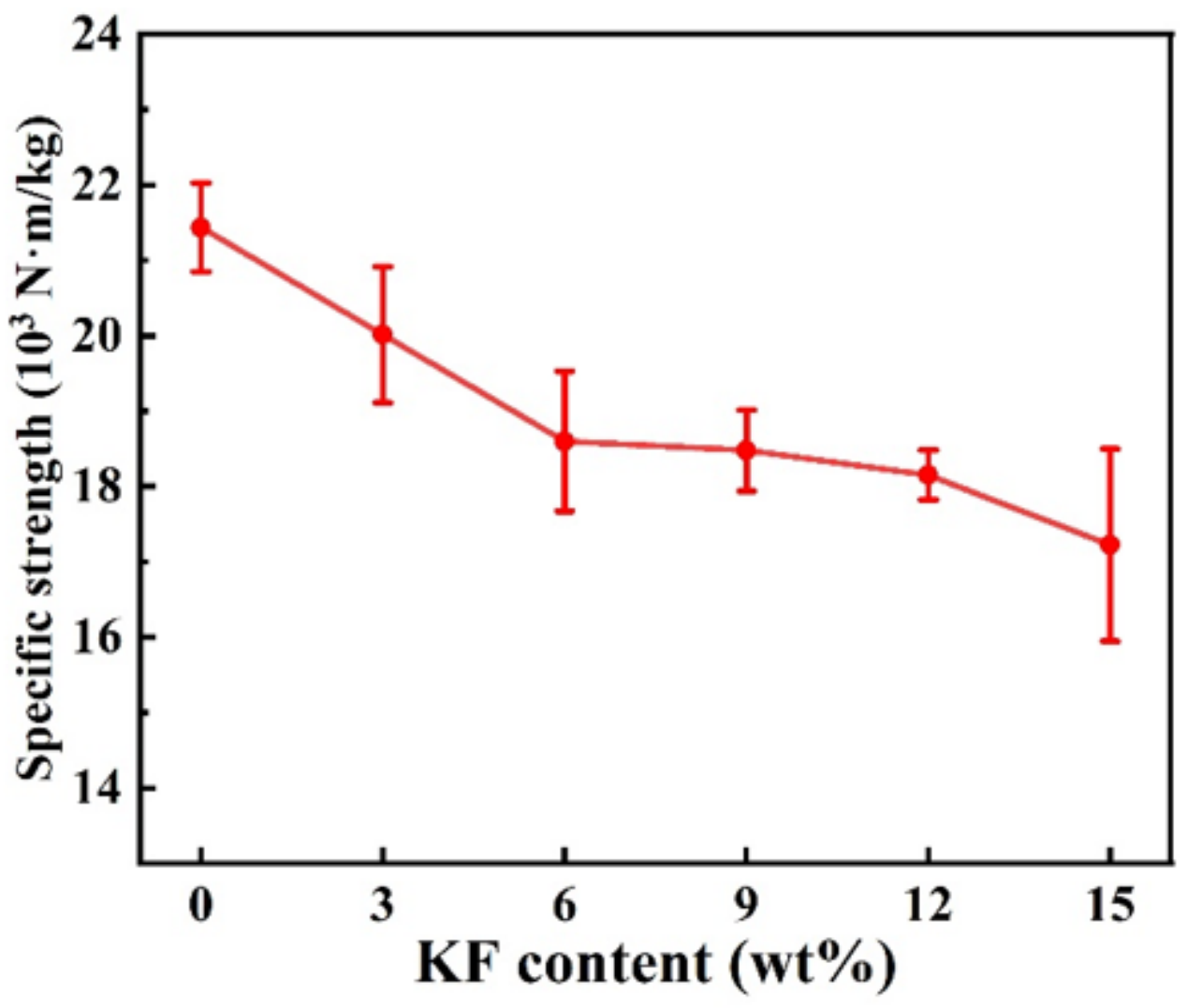

Figure 5 
Comparison of specific strength of the PVDF/KF with different KF content.
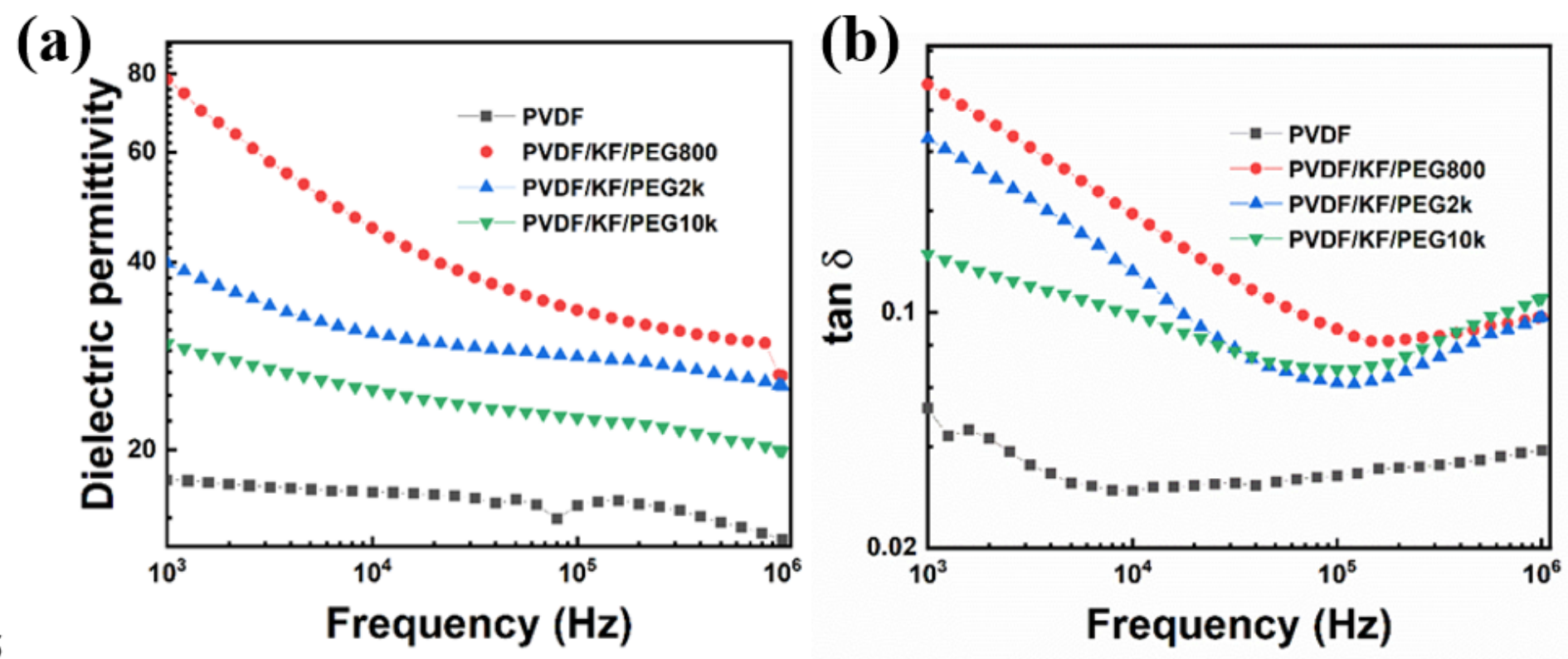

Figure 6

Dielectric constant (a) and dielectric loss tangent (b) versus frequency for the PVDF and PVDF/KF/PEG containing PEG with various molecular weights. 


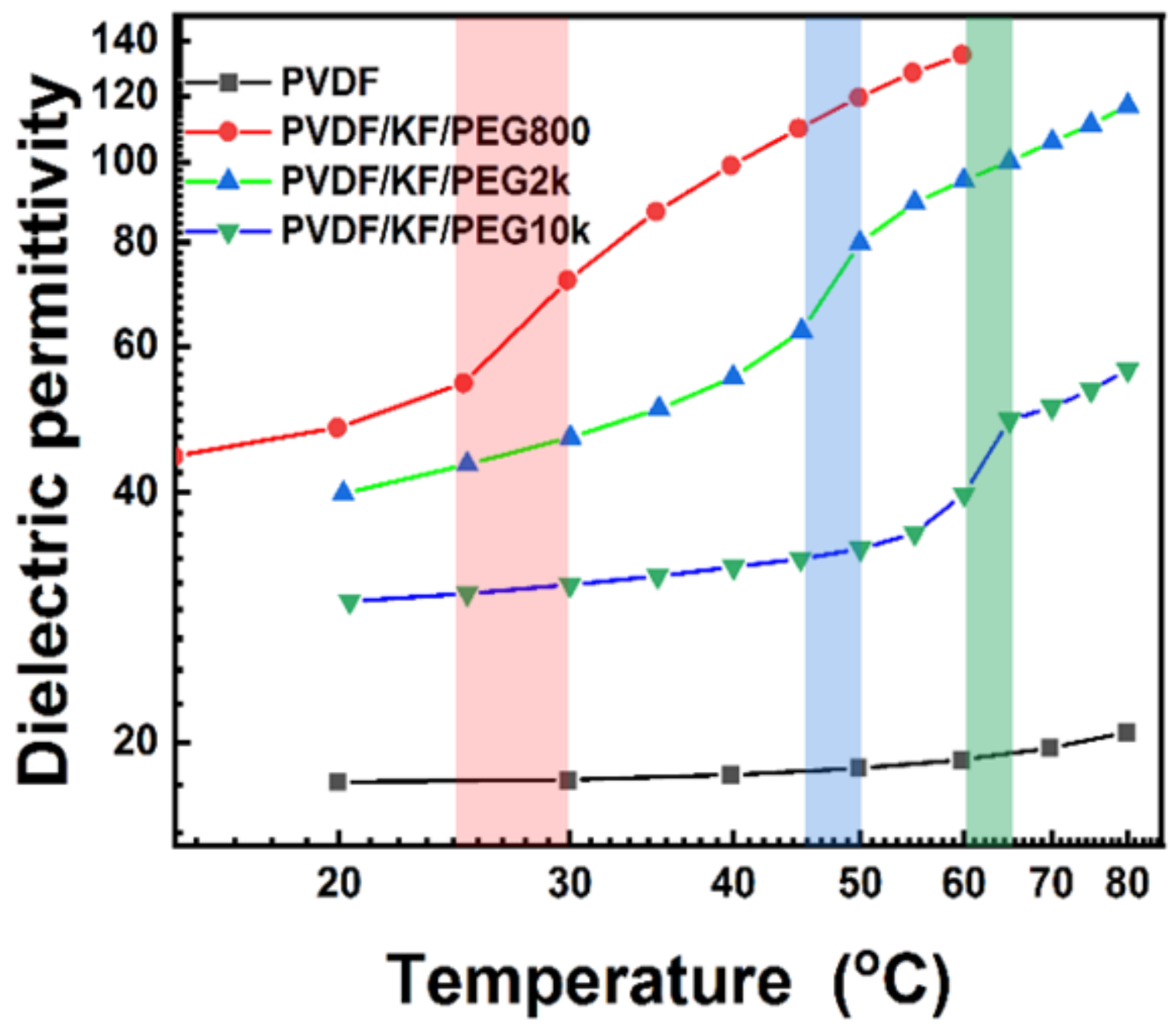

Figure 7

Temperature dependence of the dielectric constants of the pure PVDF and PVDF/KF/PEG composite films.

(a)

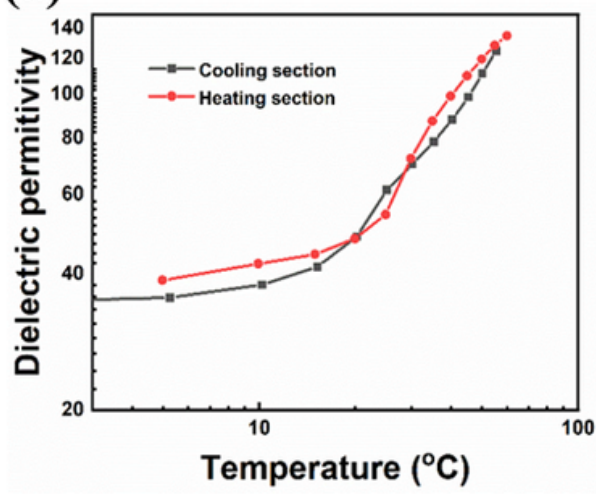

(b)

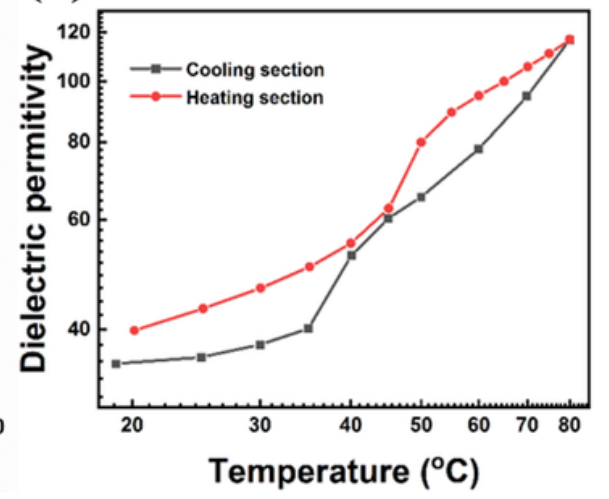

(c)

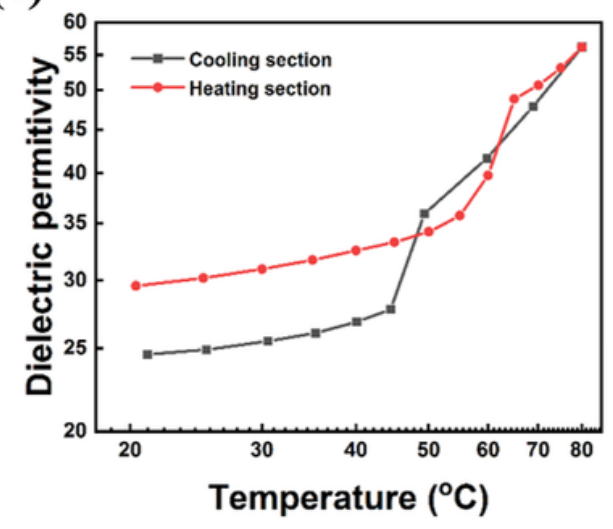

Figure 8 
Thermal hysteresis loop of the PVDF/KF/PEG800 (a), PVDF/KF/PEG2k (b) and PVDF/KF/PEG10k (c) composites at $1 \mathrm{kHz}$.

\section{Supplementary Files}

This is a list of supplementary files associated with this preprint. Click to download.

- GraphicalAbstract.png

- Supportingmaterials.docx 